\title{
1 Exhaustion and Inflation at Antipodes of T-cell Responses to Chronic Virus Infection
}

3 Luka Cicin-Sain ${ }^{1,2,3}$, Ramon Arens ${ }^{4}$

$4{ }^{1}$ Department of Vaccinology and Applied Microbiology, Helmholtz Centre for Infection

5 Research, Braunschweig, Germany

$6 \quad{ }^{2}$ Institute for Virology, Medical School Hannover, Hannover, Germany

$7 \quad{ }^{3}$ German Center for Infection Research (DZIF), Partner site Hannover/Braunschweig, Germany

$8{ }^{4}$ Department of Immunohematology and Blood Transfusion, Leiden University Medical Center,

9 Leiden, the Netherlands

10 Correspondence: Luka.cicin-sain@helmholtz-hzi.de

11 Keywords: Immune Exhaustion; Memory Inflation; Chronic Virus Infection; Viral Latency; Viral

12 Persistence; CD8 T-cell

\section{Abstract}

14 Viruses that have coevolved with their host establish chronic infections that are well tolerated by 15 the host. Other viruses, that are partly adapted to their host, may induce chronic infections where persistent replication and viral antigen expression occur. The former induce highly functional and resilient CD8 T-cell responses called memory inflation. The latter induce dysfunctional and exhausted responses. The reasons compelling T-cell responses towards inflationary or exhausted responses are only partly understood. In this review we compare the two conditions and describe mechanistic similarities and differences. We also provide a list of potential reasons why

21 exhaustion or inflation occur in different virus infections. We propose that $\mathrm{T}$ cell mediated

22 transcriptional repression of viral gene expression provides a critical feature of inflation that

23 allows peaceful virus and host coexistence. The virus is controlled, but its genome is not

24 eradicated. If this mechanism is not available, as in the case of RNA viruses, the virus and the host are compelled to an arms race. If virus proliferation and spread proceed uncontrolled for too

26 long, $\mathrm{T}$ cells are forced to strike a balance between viral control and tissue destruction, losing

27 antiviral potency and facilitating virus persistence. 
29 Viruses critically depend on the host for their survival and reproduction. This lifestyle compels

30 the viruses to a continuous dance on a knife-edge. On one hand, they are relentlessly hunted by

31 the immune system; they may propagate only if they avoid detection or outrun the host defenses.

32 Yet, even if they succeed at overwhelming the immunity, this is likely to result in disease and

33 death of the host, and thus ultimately in the demise of the virus.

34 Natural selection has forced viruses to either aggressively transmit through large and diverse

35 populations of hosts [1], or to undergo co-evolution with defined host species and coexist over

36 long-periods of time minimizing the harm to the host [2]. The first strategy is manifest in viruses

37 that rapidly evolve to cause infections across various species (such as influenza virus), or in

38 vector-carried viruses whose transmission is not hindered by severe disease that immobilizes the

39 host (such as arboviruses) [1]. The outcome of such infections may be the resolution of the

40 disease and the clearance of infection, or disease progression until death. Viral survival is

41 achieved by their rapid spread to other hosts. On the other hand, viruses that are well adapted to

42 the host typically have milder disease courses and establish a détente with the host immune

43 system. This allows viral persistence in absence of overt disease for long periods, but requires

44 viral adaptation to the specific immune system of the host. These outcomes are common in

45 herpesvirus infections, where the persistence of viral genomes in host cells is achieved by

46 silencing the transcription of most of their genes. The latently infected host is typically healthy

47 and unaware of the presence of the latent virus in the body, yet the virus may reactivate if the

48 host becomes severely ill [3], providing a chance to the virus to spread from the dying host.

49 Wide varieties of outcomes are possible between these two extremes. Among those intermediate

50 outcomes, are the clinically relevant persistent virus infections. Human immunodeficiency virus 2 
51 (HIV), hepatitis C virus (HCV), and to a lesser extent hepatitis B virus (HBV) cause only mild

52 direct cytopathic effects and thus may persistently proliferate in hosts, driving chronic and 53 progressive diseases that are fatal unless treated. These remain a major public health burden

54 worldwide, despite tremendous advances in therapeutic options against HCV and HIV. For

55 instance, a therapy clearing HIV from the body is still missing. Therefore, there is a growing 56 population of patients worldwide who undergo combined retroviral therapy over numerous years,

57 where HIV persistence encumbers the immune system, thus increasing the risk of inflammatory

58 conditions and cancer, as well as accelerating the onset of immune aging $[4,5]$.

59 Since virus chronicity requires adaptation to the host, clinically relevant chronic viruses are 60 highly adapted to humans and cannot be efficiently studied in animal models in vivo. Therefore, 61 experimental models of immune responses to persistent or latent infections have relied on viruses 62 that naturally infect animals, and in particular mice. The most common mouse models of chronic 63 virus infection are based on the infection of mice with lymphocytic choriomeningitis virus 64 (LCMV) clone 13 [6] and with LCMV strain WE [7]. These LCMV models induce a state of 65 virus-specific T cell 'exhaustion' (as will be discussed in detail hereafter), which has remarkable 66 similarity with the $\mathrm{T}$ cell responses to chronic viral infections in humans such as HIV or hepatitis 67 C virus infections [8]. The immune response to a persistent (latent) herpesvirus has been 68 extensively studied in the mouse CMV (MCMV) infection model [9], which has striking 69 resemblance to HCMV infection with respect to the impact on the function and phenotype of the 70 virus-specific $\mathrm{T}$ cells [10], or to $\mathrm{T}$ cell responses to other DNA-virus infections, such as 71 adenoviruses $[11,12]$.

\section{Effects of chronic viral infections on the immune system}


73 Persistent infections with high-level replicating viruses, like HIV, HCV or LCMV induce T-cell

74 responses that share similar traits in mice and men. Over time, cytokine production and

75 cytotoxicity in antigen-specific CD8 T-cell populations is lost $[8,13]$. This loss of function is not

76 only associated with poor control of the offending virus, but also with an increase in chronic

77 inflammation that induces cumulative immune pathology, a propensity for cancer and a

78 premature onset of immune senescence [14, 15]. Such effects are particularly pronounced in

79 patients co-infected with HIV and $\mathrm{HCV}$, where HIV co-infection accelerates inflammatory liver

80 injuries and hepatic decompensation elicited by HCV [16].

81 It is important to note that herpesviruses, such as cytomegalovirus (CMV), have also been

82 suspected to play a role in immune senescence [17]. Ongoing and intermittent antigenic

83 stimulation by CMV engages the cellular immune system at times of latency [18, 19], driving

84 responses of differentiated T cells [20]. However, the scientific consensus has evolved over the

85 years towards a conclusion that the presence of latent CMV does not necessarily accelerate the

86 onset of immune aging or impair the immune system in older people [21, 22], but is likely linked

87 to the strength of the CMV infection and the immune status of the host.

88 It remains unclear why different chronic infections result in diametrically opposing outcomes in

89 the functionality of responding T cells. Why do some chronic virus infection exhaust the immune

90 system, while other ones do not? How may we guide the immune reaction to HCV or HIV

91 towards a functional and protective response? To begin to understand these aspects, one needs to

92 consider the specificities of the immune response to latent viruses and distinguish them from the

93 immune exhaustion elicited by productively replicating chronic-persistent infections. These two

94 scenarios will be described and reasons for their divergent outcomes discussed.

\section{Immune exhaustion}


96 Productive viral replication induces chronic inflammatory conditions and exhausts the adaptive

97 host immune system over time, in particular the CD8 T-cell compartment [19, 23]. While 98 exhaustion and virus persistence are parts of a vicious cycle, it remains unclear if the inability of 99 exhausted $\mathrm{T}$ cells to clear the virus results in persistent infection, or if viral persistence results in 100 exhaustion. Either way, these viruses pose major clinical problems, not only due to direct 101 cytotoxicity, but also due to the long-term immune pathology that they elicit. The paradigmatic 102 murine LCMV infection models allowed the study of immune responses to chronic persistent 103 infections in mechanistic detail. Labelling of $\mathrm{T}$ cells with peptide-MHC (pMHC) tetramers 104 revealed that the virus-specific T cells are not lost in chronic LCMV infection. They are merely 105 hypofunctional cells, designated as exhausted, in functional assays (e.g. cytokine production) 106 [13]. T cell exhaustion is driven by continuous high-level cognate antigenic triggering, and 107 eventually exhausted $\mathrm{T}$ cells become antigen-addicted for their maintenance $[24,25]$. In contrast, 108 conventional $\mathrm{T}$ cells do not rely on their cognate antigen for survival but on IL-7 and/or IL-15109 driven homeostatic self-renewal [26]. Comparison of transcriptional networks in LCMV-specific 110 CD8 $\mathrm{T}$ cells revealed a partial overlap of genes that are activated during acute and chronic 111 LCMV infection, and a key role for the transcriptional factors T-bet and Eomesodermin 112 (EOMES) in both conditions [27] (Table 1). Importantly, the CD28-like PD-1 receptor is retained 113 on exhausted T cells and inhibition of PD-1 with its ligand PD-L1 by monoclonal antibodies 114 restores the $\mathrm{T}$ cell function and enhances the clearance of chronic LCMV infection [28]. PD-1 115 blockade also restores the function of HIV-1 specific T cells [29, 30]. However, reprogramming 116 of exhausted T cells into durable memory T cells via blocking PD-1 is limited due to irreversible 117 epigenetic alterations [31]. Interestingly, responses to LCMV antigens are not reduced in 118 immunoproteasome deficient mice [32] and antigen presentation on non-hematopoietic cells 119 substantially expands the pool of responding T cells [33]. 
120 Besides PD-1 expression, exhausted $\mathrm{T}$ cells express a number of other inhibitory receptors 121 including CTLA-4, LAG3, TIM3, 2B4, CD160 and TIGIT [34]. Moreover, molecules involved in 122 metabolism like the ectonucleotidase CD39 are also highly expressed [35]. Expression of central123 memory markers such as CD62L and CD127 (IL-7R $\alpha$ ) is absent. Remarkably, the effector cell 124 marker KLRG1 is not highly expressed [36]. It is important to note that the exhausted state of T 125 cells is acquired progressively. For example, the loss in cytokine polyfunctionality of exhausted $126 \mathrm{CD}^{+} \mathrm{T}$ cells starts with the loss of IL-2 followed by tumor necrosis factor (TNF) and finally the 127 capacity to produce interferon-gamma (IFN $\gamma$ ) wanes. The progressive loss of memory $\mathrm{CD}^{+} \mathrm{T}$ 128 cell potential is likely associated with the gradual loss of autocrine IL-2 production [37]. Notably, 129 heterogeneity exists in exhausted T cell populations. Exhausted T cells can be reinvigorated by 130 blocking PD-1 and other inhibitory receptors, but cells expressing high levels of T-bet and 131 intermediate PD-1 expression respond better to PD-1 blockade as compared to cells expressing 132 high levels of PD-1 and EOMES [38, 39]. Either way, this reversion of the exhausted state has 133 led to clear clinical benefit in chronically infected individuals and cancer patients, arguing that 134 exhausted cells are essentially dysfunctional. While exhaustion may represent a breakdown of the 135 equilibrium between the immune system and a persistent virus, it has recently been proposed that 136 repressed functionality in "exhausted" CD8 T cells serves to limit immune pathogenesis, while 137 the same CD8 T cells still contribute to immune surveillance of the virus [40]. This idea was 138 predicated on observations that persistent viruses rapidly replicate in animals lacking CD8 T cells 139 and that exhausted phenotypes are observed in patients with good outcomes of autoimmune 140 disease [41]. In that case, exhaustion might be a misnomer, because "exhausted" CD8 T cells 141 contribute to host survival. Therefore, it is possible that exhaustion is a condition of equilibrium 142 after all.

\section{$143 \frac{\text { Memory Inflation }}{6}$}


144 Ongoing antigenic stimulation by latent herpesviruses, in particular by the $\beta$-herpesvirus CMV, 145 also strongly engages the cellular immune system in the chronic phase of infection [18, 42]. 146 However, the functionality of CMV-specific T-cells is maintained into old age [22, 43], and 147 functional responses to in vivo CMV challenge in immunosenescent non-human primates were 148 essentially undistinguishable from those in young adult monkeys [44]. T-cell depletions in 149 experimentally infected animals showed that persistent T-cell responses, and in particular a 150 functional IFN $\gamma$ response, are crucial for the repression of CMV reactivation from latency [45]. 151 This life-long functionality of T-cell responses is particularly remarkable in light of T-cell 152 exhaustion in other scenarios of virus persistence [46]. Hence, juxtaposing the processes 153 underlying T-cell responses to CMV and exhausted responses to persistent viruses may help us to 154 understand both of these mechanisms. In this respect, it is noteworthy to mention that the 155 transcriptional signatures of inflationary $\mathrm{CD} 8 \mathrm{~T}$ cells are different from conventional or 156 exhausted $\mathrm{T}$ cells with respect to the level of transcription factors such as Blimp1, T-bet and 157 EOMES (Table 1).

158 It is important to note that Rhesus CMV (RhCMV) based vaccine vectors may elicit highly 159 unconventional CD8 T-cell responses against epitopes presented on MHC-II [47] or HLA-E 160 molecules [48]. However, this was shown to occur only in the context of a RhCMV mutant that 161 was cloned upon extensive in vitro passaging of the virus [49]. This does not seem to represent 162 the response of human CMV (HCMV)-based vaccines [50], or natural T-cell responses to wild163 type RhCMV infection, which elicits conventional MHC-I restricted CD8 T-cell responses [47, 164 48]. Therefore, the nature of the RhCMV vector-induced responses will not be discussed further.

165 The ongoing accumulation of antigen-specific CD8 $\mathrm{T}$ cells in CMV infection has been first 166 described in the mouse model [51] and aptly termed memory inflation [52] (reviewed in [42]). 
167 Data from the murine model closely predicted the nature of CD8 T-cell responses to human CMV

$168[53,54]$. The inflationary responses accrue over time, but do not constitute an overall expansion

169 of the primed compartment, whose size remains relatively stable upon infection [55]. Rather,

170 some antigenic epitopes encoded by MCMV induce dominant inflationary responses and expand

171 at the expense of other, subdominant, epitopes $[56,57]$. The phenotype of inflationary $\mathrm{T}$ cells is

172 effector-memory like, and is characterized by low levels of CD62L and CD127. In contrast to

173 exhausted T cells, KLRG1 is highly expressed, while PD-1 expression is low [58, 59]. Other

174 epitopes elicit conventional immune responses, akin to responses observed upon infection with

175 non-persistent pathogens. These responses are marked by robust expansion of T cells early upon

176 infection, contraction by day 14 and a shift of phenotypes of antigen-specific $\mathrm{T}$ cells towards

177 central-memory like during the maintenance phase $[56,60]$. Therefore, the requirements for

178 inflation can be studied by comparing conventional or inflationary CD8 T-cell responses in the

179 context of MCMV infection. In this respect, it should be noted that memory inflation is not

180 exclusively linked to CMV infection (albeit most pronounced), but is also observed after

181 infection with certain adenovirus and parvovirus strains [59].

182 Inflationary responses require the presentation of antigenic epitopes on non-hematopoietic cells, 183 whereas this is dispensable for conventional responses [61, 62]. On the other hand, conventional 184 responses require processing by the immunoproteasome, yet the constitutive proteasome is 185 sufficient for the emergence of inflationary responses [63]. We showed recently that moving an 186 immunoproteasome-dependent MCMV epitope from its native position within the viral protein to 187 an alternative position where the epitope is available to processing by the constitutive proteasome 188 resulted in drastic changes in size and quality of responses [64]. The response improved by a 189 factor of 10, shifted from conventional to inflationary, and was present in mice with impaired 190 antigen presentation on hematopoietic cells [64]. Taken together, these data demonstrated that 8 
191 antigen processed by the constitutional proteasome in non-hematopoietic cells sustains 192 inflationary responses during virus latency.

193 So, why do non-hematopoietic cells drive inflationary responses? Although this question is not 194 conclusively answered, it is likely that this depends on the cells that harbor latent MCMV (and 195 thus that express viral antigens at times of latency). MCMV transcription proceeds at low levels 196 during virus latency [65] and endothelial cells were shown to harbor latent virus [66]. The link 197 between latent transcription and memory inflation was exposed by a study where latent 198 transcription of viral genes was enhanced in an MCMV mutant lacking a single antigenic epitope 199 within the IE1 gene [67]. It has been therefore proposed that low levels of sporadic antigenic 200 expression in latent CMV infection drive CD8 T-cell responses, which in turn limits further viral 201 transcription, establishing a state of dynamic equilibrium between the virus and the host [53]. 202 This theory, called Immune Sensing theory, has been further corroborated by transgenic MCMVs 203 expressing foreign epitopes. These epitopes induced forceful inflationary T-cell responses, yet the 204 inflationary response against endogenous epitopes was significantly diminished [68, 69] at the 205 expense of effector cell responses, while central memory responses against the same epitopes 206 were unaffected [70]. The competition of antigenic peptides for inflationary CD8 T-cell 207 responses was not observed when mice were coinfected with the mutant and the wild-type 208 MCMV [69]. This behavior was predicted by the Immune Sensing theory [53], because CD8 T 209 cells would only be able to compete for epitopes that are expressed within the same latently 210 infected cell. If viral genes were expressed from different cells, the dominant epitope could not 211 outcompete the subdominant ones. At a glance, this theory contradicts clinical evidence that the 212 immunodominant and inflating CD8 T-cell response to the HLA A2:01 restricted HCMV epitope 213 NLVPMVATV [71, 72] targets a peptide derived from a late HCMV gene (i.e. UL83 (pp65)), 214 because immune sensing should prevent the expression of late genes. However, HCMV is 9 
215 maintained latent in numerous cell types, including hematopoietic cells [73], yet the

216 transcriptional activity of UL83 was assessed in fibroblastic cell cultures, rather than primary

217 human cells bearing latent genomes. Thus, additional evidence is required to understand how

218 UL83/pp65 immunodominance may fit into the immune sensing theory (or alternatively, how it 219 may disprove it).

220 Taken together, a model emerges where the non-hematopoietic cells transcribe low levels of

221 antigen from otherwise latent CMVs, and thus keep poking CD8 T cells. CD8 T cells respond 222 with IFN $\gamma$ production, which represses viral transcription, and reaffirms the latent state, thus

223 providing relief to the $\mathrm{T}$ cells. The epitopes that induce such responses are processed by the 224 constitutive proteasome, which means that epitope presentation does not require interferon225 mediated induction of the proteasome. This then implies that viral control is achieved in 226 conditions of minimal inflammation. Such balance hinges on transcriptional silencing of viral 227 DNA (Fig.1) by cytokines secreted by T cells. Notably, viruses inducing T-cell exhaustion in 228 chronic infection are typically RNA viruses, and thus may not allow a similar peaceful 229 coexistence with the host.

\section{Potential causes of differences between inflation and exhaustion}

231 CD8 T-cell responses to chronic LCMV and MCMV differ in their functional capacity, but 232 notably also display numerous similarities. The priming of naïve virus-specific CD8 T cells does 233 not occur exclusively during primary LCMV or MCMV infection, since novel naïve cells are also 234 recruited in the chronic phase $[58,74,75]$. T-cell responses to LCMV-encoded antigens [32] or 235 inflationary MCMV epitopes are still maintained in immunoproteasome deficient mice [63]. The 236 pool of responding $\mathrm{T}$ cells is substantially expanded by antigen-presentation on non237 hematopoietic cells upon MCMV or acute LCMV infection [33, 61], and antigen presentation on 10 
238 the non-hematopoietic cells exacerbates exhaustion in chronic LCMV infection [76]. Likewise,

239 virus specific cells showing effector phenotypes (KLRG1 $\left.{ }^{+}, \mathrm{CD}^{2} \mathrm{~L}^{-}, \mathrm{CD}^{-} 7^{-}, \mathrm{CD}_{127^{-}}\right)$indicative

240 of recent antigenic encounter, are detected for a long time after either of these infections. The

241 responding cells seem to depend on continuous TCR stimulation to maintain their pools, as

242 evidenced in adoptive transfer experiments $[25,58]$. Therefore, it stands to reason that antigens

243 are expressed during LCMV persistence, but also during CMV latency. In light of that, we

244 consider several models that might explain the difference in T-cell functionality in these two

245 scenarios. Notably, these propositions are not mutually exclusive, and it is likely that two or more

246 occur at the same time and are interconnected.

Antigen persistence vs. intermittence

It has been proposed that virus replication and thus antigenic stimulation causes immune

exhaustion while intermittent virus replication with limited periods of antigen presence would

250 retain functional T-cell responses [19]. It is important to consider that herpesviruses typically

251 cause productive infections that lyse the infected cells [77]. Therefore, antigen expression during

252 CMV latency is bound to be a result of intermittent and recurring transcriptional events [78],

253 rather than an ongoing and continuous production. On the other hand, the direct cytopathic effect

254 of hepatitis viruses is typically low [79], implying that productive hepatitis infections may

255 simmer continuously. A similar persistence of antigenic expression was described in LCMV

256 variants associated with immune exhaustion [80]. Hence, MCMV and LCMV infections may

257 induce different kinds of T-cell responses due to the intermittent antigen expression in MCMV

258 infection, which differs from continuous and sustained presence of an antigen in LCMV

259 infection. However, this explanation is essentially based on correlative evidence. Therefore, it

260 remains unclear if forced persistence of an antigen in the context of an MCMV infection would 
261 also drive the exhaustion of cognate T cells. Furthermore, exhaustion and inflation alone do not 262 explain why some viruses replicate persistently, whereas other ones only intermittently.

264 Another proposed explanation for the onset of exhaustion is that strong viral replication during 265 the onset of virus infection promotes viral persistence and drives exhaustion [80]. [81]. This idea

266 fits with experimental evidence that the abundance and availability of LCMV antigen defines the 267 extent of exhaustion [76, 82]. The conditions of primary MCMV infection also define the latent 268 virus load [83] and the size of the inflationary response [84], but the amount of antigen remains 269 overall low since virus replication is essentially silenced [85], and limited virus antigen is present

270 in conditions of MCMV inflation [65, 78]. Consequently, persistent antigen leading to exhaustion 271 is much more abundant than the antigen driving inflationary phenotypes. On the other hand, this 272 interpretation is likely to be too simplistic, because it would imply that a low-dose infection with

273 LCMV or hepatitis $\mathrm{C}$ would result in inflationary responses, but clinical and experimental

274 evidence argues that low dose infection with these viruses results in virus clearance and 275 conventional responses.

277 It has also been proposed that the cellular niche of viral replication predisposes the immune 278 response towards exhaustion [86]. Both MCMV and LCMV persistently stimulate CD8 T-cell 279 responses by antigen expression in non-hematopoietic cells (see above), but MCMV is latent in 280 liver endothelial cells [66], while LCMV persists in fibroblastic reticular cells [87]. However, it is 281 unclear if either virus is restricted to these cell types during the chronic phase of infection, or may 
282 be found in other ones as well. Furthermore, a mechanism explaining the link between virus

283 tropism for defined cell types and T-cell responses has not been established.

285 We compared the priming of CD8 T-cell responses upon primary LCMV or MCMV infection 286 and observed a clear difference in costimulatory signal ("signal 2") requirement [88]. We 287 analyzed responses to the LCMV epitope KAVYNFATC $\left(\mathrm{GP}^{33}\right)$ upon infection with LCMV or a recombinant MCMV expressing the same epitope and showed that co-stimulation by CD80/86 is required for priming against $\mathrm{GP}^{33}$ when expressed by $\mathrm{MCMV}$, but highly redundant with other 290 signal 2 co-receptors in the context of LCMV infection. On the other hand, LCMV infection 291 induced much stronger type I IFN responses. Soluble cytokines may co-stimulate T cells during 292 priming ("signal 3") and priming against LCMV-encoded epitopes depended strongly on type I 293 IFN-dependent signal 3 [88]. CD8 T-cells lacking type I IFN receptors are susceptible to NK294 cell mediated apoptosis in chronic LCMV infection [89, 90], implying that IFN- $\alpha / \beta$ provides a critical survival signal to these cells. However, it remains unclear if CD8 T-cell survival depends on strong type I IFN responses to LCMV, or if tonic IFN responses would be sufficient. While 297 high levels of type I IFN promote T-cell priming [88] in acute LCMV infection, they were also 298 shown to support the onset of chronic virus infection [91, 92]. IL-12 and type I IFN responses are 299 more balanced during MCMV infection, but type I IFN responses push MCMV into latency [93], 300 implying that interferon induces the chronic state in both infections and that exhaustion or 301 inflation pathways might be defined during the priming stage of $\mathrm{T}$ cells. While we observed 302 clearly different priming requirements in MCMV and acute LCMV infections [88], a comparison 303 of T-cell responses to $\mathrm{GP}^{33}$ expressed by LCMV or the recombinant MCMV during chronic 
304 infection has not yet been performed. Therefore, further studies are required to address this 305 question conclusively.

In LCMV infection, the progressive exhaustion of CD8 $\mathrm{T}$ cells is accelerated by the lack of CD4 $308 \mathrm{~T}$ cells $[94,95]$. Moreover, high dose infection has been shown to result in the deletion of 309 activated CD4 T-cells by activated NK cells, thereby promoting exhaustion [96]. Taken together, 310 a relative lack of CD4 $\mathrm{T}$ cells promotes exhaustion. In MCMV infection, the development of

311 inflationary $\mathrm{CD} 8^{+} \mathrm{T}$ cells depends on the presence of CD4 T cells [97]. This feature affects only 312 some inflationary epitopes [98], although this requirement was much stricter upon infection with 313 a viral mutant that is poorly controlled by NK cells [99]. CD4 T cells may affect virus replication 314 directly by releasing antiviral cytokines or indirectly by affecting CD8 T cells or B cells. For 315 instance, interleukin 10 (IL-10), a cytokine that is frequently released by regulatory CD4 $\mathrm{T}$ cells 316 affects memory inflation, which was much more pronounced in IL-10 deficient mice [100]. On 317 the other hand, the modulation of $\mathrm{T}$ cell activity by regulatory CD4 T cells exerts pleiotropic and 318 organ specific effects in the spleen and salivary glands [101]. Therefore, these phenomena are 319 complex and complicated, and the net effect of CD4 T cells remains incompletely understood.

The common cytokine receptor gamma chain $\left(\gamma_{c}\right)$ cytokines $I L-2, I L-7$, and $I L-15$.

322 The common $\gamma_{\mathrm{c}}$ cytokine family members have crucial roles in T-cell survival, proliferation and 323 differentiation [102]. IL-2 signaling involving CD25 (IL-2R $\alpha$ : forming together with CD122 and 324 CD132 the high-affinity IL-2R complex) is important for the maintenance of both exhausted and 325 inflationary $\mathrm{CD} 8^{+} \mathrm{T}$ cells [103]. Since the percentage of cells producing autocrine IL-2 within the 
326 inflationary $\mathrm{T}$ cell populations correlates to their expansion of the inflationary pool [104], the

327 induction of autocrine IL-2 appears to be critical for inflationary expansions. Remarkably, the

328 expression of CD122, the IL-2R $\beta$ chain, which is also shared by IL-15, is differentially

329 expressed. Inflationary $\mathrm{CD}^{+} \mathrm{T}$ cells have low CD122 levels [58, 60], while exhausted cells

330 maintain CD122 expression. The increased expression of CD122 marks the exhausted state, and

331 signaling via CD122 upon IL-2 and IL-15 binding is likely directly involved via upregulation of

332 inhibitory receptors [105]. Both exhausted and inflationary T cells have low CD127 (IL-7R $\alpha$ )

333 expression. However, long-term IL-7 treatment during the contraction phase of chronic LCMV

334 infection enhances the magnitude and functionality of specific CD8 ${ }^{+} \mathrm{T}$ cells $[106,107]$.

335 Ag transcriptional repression vs. repression by killing of infected cells

336 Type I and II IFNs play an important role in the control of MCMV infection; in IFN- $\gamma \mathrm{R}^{-/-}$mice 337 MCMV replicates persistently [108]. Similarly, type I IFN represses viral gene expression by 338 upregulating nuclear domain 10 (ND10) proteins in a reversible process [93]. While type I and 339 type II IFN signaling was shown to limit LCMV replication as well [109, 110], there is no 340 evidence that this repression may be transient. The IFN induced silencing of DNA viruses can 341 affect any episomal DNA within the cell nucleus and silence their transcription [93]. It is 342 therefore reasonable to assume that IFN- $\gamma$ signaling may silence the transcription of DNA viruses 343 and represses the expression of antigens upon T-cell activation. Interestingly, immune exhaustion 344 is typically induced by RNA viruses, where IFN-dependent silencing of DNA transcription 345 cannot limit antigenic expression. In that case, the ability to limit virus persistence by 346 transcriptional repression, rather than by cytotoxicity may present a critical hallmark of 347 inflationary responses, distinguishing it from events in immune exhaustion. Therefore, we 348 hypothesize that the cytotoxic activity of T cells may compel persistent RNA viruses to an arms 
race, where they rapidly replicate to achieve escape velocity from T-cell control. T-cell

350 proliferation upon activation is uniquely rapid with a 2-hour cell cycle time [111]. However, a

351 replicating virus gives rise to thousands of infectious particles per lytic cycle, spurring drastic

352 expansions of responsive CD8 T-cell clones. If T cells are unable to limit the spread of a rapidly

353 replicating virus, cytotoxicity itself will become detrimental to the host. We postulate that such

354 potential for immune pathology is sensed during the immune response and that the immune

355 exhaustion program sets in to protect the host. While this hypothesis is consistent with published

356 data, further detailed studies will be required to validate our prediction.

\section{Concluding remarks}

358 The characterization of exhausted versus inflationary T-cell responses in chronic viral infections

359 is advancing in great detail. Available evidence indicates that both inflation and exhaustion are

360 conditions of equilibrium between the host and the persisting virus, yet their clinical outcomes

361 are vastly different, because they depend on distinct cellular and molecular mechanisms. While

362 thorough understanding of the underlying mechanisms leading to these divergent cellular states

363 remains lacking, the targeting of inhibitory pathways of exhausted T cells has significantly

364 innovated immunotherapy of chronic infection and cancer, and exploiting of inflationary

365 responses to improve vaccines has great potential. Addressing the outstanding questions (see

366 Outstanding Questions Box) will allow manipulations of the antigenic supply and costimulatory

367 molecules that will allow the induction of optimal and protective T-cell responses.

\section{Acknowledgments}

369 This work was supported by the ERC-POC grant VIVAVE and the DFG grant (SFB900 TP B2)

370 to LCS, and a Dutch Cancer Society grant (KWF UL2015-7817) awarded to RA. 


\section{$371 \quad$ Figure legends}

372 Figure 1: Model of T-cell mediated control of viral infections by IFN signaling or cytotoxicity. 373 Antigenic peptides presented on MHC-I molecules (pMHC-I) are recognized by inflationary or 374 exhausted CD8 T-cells. Cytokines regulating the transcription of viral genes may repress gene 375 expression in the case of DNA viruses whose genomes are maintained in the cell nucleus. This 376 non-lethal control is not available to RNA viruses, which are controlled by cytotoxic mechanisms

377 (e.g. perforin and GzB). Therefore, they are unable to establish an equilibrium with the host at the 378 level of single infected cells. This in turn compels RNA viruses to rapid proliferation to 379 overcome host control. We propose that the difference in the surface receptor expression on 380 inflationary and exhausted CD8 T-cells may be a result of continuous stimulation with large 381 amounts of antigen, as opposed to the intermittent exposure to low-levels of antigen. 


\begin{tabular}{|c|c|c|c|}
\hline \multicolumn{4}{|c|}{ Table 1. Comparison of viral-specific CD8 T-cell populations ${ }^{a}$} \\
\hline & Central-memory & Inflationary & Exhausted \\
\hline \multirow[t]{2}{*}{ Homeostatic proliferation } & ++ & - & - \\
\hline & & & \\
\hline \multirow[t]{2}{*}{ Antigen-dependence } & - & ++ & ++ \\
\hline & & & \\
\hline \multirow[t]{2}{*}{ 2e Expansion capacity } & ++ & $+/-$ & - \\
\hline & & & \\
\hline \multirow[t]{2}{*}{ Cytokine polyfunctionality } & ++ & + (low \% IL-2) & - \\
\hline & & & \\
\hline \multicolumn{4}{|l|}{ Lymphoid homing markers } \\
\hline CD62L & ++ & - & - \\
\hline \multirow[t]{2}{*}{ CCR7 } & ++ & - & - \\
\hline & & & \\
\hline \multicolumn{4}{|l|}{ Cytokine receptors } \\
\hline CD122 & ++ & - & + \\
\hline \multirow[t]{2}{*}{ CD127 } & ++ & $+/-$ & - \\
\hline & & & \\
\hline \multicolumn{4}{|l|}{ NK cell receptors } \\
\hline \multirow[t]{2}{*}{ KLRG1 } & - & ++ & - \\
\hline & \multicolumn{2}{|c|}{ Costimulatory receptors } & \\
\hline $\mathrm{CD} 28$ & + & - & - \\
\hline \multirow{2}{*}{ CD27 } & + & - & - \\
\hline & & & \\
\hline \multicolumn{4}{|l|}{ Inhibitory receptors } \\
\hline \multirow[t]{2}{*}{ PD-1/TIM3/LAG3/ etc. } & - & - & ++ \\
\hline & & & \\
\hline \multicolumn{4}{|l|}{ Transcription factors } \\
\hline T-bet & - & + & $+/-$ \\
\hline EOMES & - & $+/-$ & + \\
\hline Blimp-1 & $+/-$ & $+/-$ & + \\
\hline
\end{tabular}

416 a - absent or low, $+/$ - intermediate, + high, ++ prominent

417 
1. Ewald, P.W. (1996) Guarding against the most dangerous emerging pathogens. Emerg Infect Dis 2 (4), 245-57.

2. Alizon, S. et al. (2009) Virulence evolution and the trade-off hypothesis: history, current state of affairs and the future. J Evol Biol 22 (2), 245-59.

423 3. Limaye, A.P. et al. (2008) Cytomegalovirus reactivation in critically ill immunocompetent patients.

424 Jama $300(4), 413-22$.

4. Appay, V. et al. (2007) Accelerated immune senescence and HIV-1 infection. Exp Gerontol 42 (5), $432-$ 7.

5. Effros, R.B. et al. (2008) Workshop on HIV Infection and Aging: What Is Known and Future Research Directions. Clinical infectious diseases : an official publication of the Infectious Diseases Society of America 47 (4), 542-553.

6. Ahmed, R. and Oldstone, M.B. (1988) Organ-specific selection of viral variants during chronic infection. J Exp Med 167 (5), 1719-24.

7. Rivers, T.M. and McNair Scott, T.F. (1935) MENINGITIS IN MAN CAUSED BY A FILTERABLE VIRUS. Science 81 (2105), 439-440.

8. Gruener, N.H. et al. (2001) Sustained dysfunction of antiviral CD8+ T lymphocytes after infection with hepatitis C virus. J Virol 75 (12), 5550-8.

9. Reddehase, M.J. et al. (2008) Murine model of cytomegalovirus latency and reactivation. Curr Top Microbiol Immunol 325, 315-31.

10. Hertoghs, K.M. et al. (2010) Molecular profiling of cytomegalovirus-induced human CD8+ T cell differentiation. J Clin Invest 120 (11), 4077-90.

11. Bolinger, B. et al. (2015) Adenoviral Vector Vaccination Induces a Conserved Program of CD8(+) T Cell Memory Differentiation in Mouse and Man. Cell Rep 13 (8), 1578-88.

12. Colston, J.M. et al. (2016) Modification of Antigen Impacts on Memory Quality after Adenovirus Vaccination. J Immunol 196 (8), 3354-63.

13. Zajac, A.J. et al. (1998) Viral immune evasion due to persistence of activated T cells without effector function. J Exp Med 188 (12), 2205-13.

14. Tu, T. et al. (2017) Chronic viral hepatitis and its association with liver cancer. Biol Chem 398 (8), 817837.

15. Naggie, S. (2017) Hepatitis C Virus, Inflammation, and Cellular Aging: Turning Back Time. Top Antivir Med 25 (1), 3-6.

16. Lo Re, V., 3rd et al. (2014) Hepatic decompensation in antiretroviral-treated patients co-infected with HIV and hepatitis $C$ virus compared with hepatitis $C$ virus-monoinfected patients: a cohort study. Ann Intern Med 160 (6), 369-79.

17. Pawelec, G. et al. (2004) Is immunosenescence infectious? Trends Immunol 25 (8), 406-10.

18. Sylwester, A.W. et al. (2005) Broadly targeted human cytomegalovirus-specific CD4+ and CD8+ T cells dominate the memory compartments of exposed subjects. J Exp Med 202 (5), 673-85.

19. Nikolich-Zugich, J. (2008) Ageing and life-long maintenance of T-cell subsets in the face of latent persistent infections. Nat Rev Immunol 8 (7), 512-22.

20. Appay, V. et al. (2002) Memory CD8+ T cells vary in differentiation phenotype in different persistent virus infections. Nat Med 8 (4), 379-85.

21. Arens, R. et al. (2015) 5(th) International Workshop on CMV and Immunosenescence - A shadow of cytomegalovirus infection on immunological memory. Eur J Immunol 45 (4), 954-7.

22. Jackson, S.E. et al. (2017) Latent Cytomegalovirus (CMV) Infection Does Not Detrimentally Alter T Cell Responses in the Healthy Old, But Increased Latent CMV Carriage Is Related to Expanded CMV-Specific T Cells. Frontiers in Immunology 8 (733). 
465

466

467

468

469

470

471

472

473

474

475

476

477

478

479

480

481

482

483

484

485

486

487

488

489

490

491

492

493

494

495

496

497

498

499

500

501

502

503

504

505

506

507

508

509

510

511

512

513

23. Virgin, H.W. et al. (2009) Redefining Chronic Viral Infection. Cell 138 (1), 30-50.

24. Shin, H. et al. (2007) Viral antigen and extensive division maintain virus-specific CD8 T cells during chronic infection. J Exp Med 204 (4), 941-9.

25. Wherry, E.J. et al. (2004) Antigen-independent memory CD8 T cells do not develop during chronic viral infection. Proc Natl Acad Sci U S A 101 (45), 16004-9.

26. Surh, C.D. and Sprent, J. (2008) Homeostasis of naive and memory T cells. Immunity 29 (6), 848-62.

27. Doering, T.A. et al. (2012) Network analysis reveals centrally connected genes and pathways involved in CD8+ T cell exhaustion versus memory. Immunity 37 (6), 1130-44.

28. Barber, D.L. et al. (2006) Restoring function in exhausted CD8 T cells during chronic viral infection. Nature 439 (7077), 682-7.

29. Day, C.L. et al. (2006) PD-1 expression on HIV-specific T cells is associated with T-cell exhaustion and disease progression. Nature 443 (7109), 350-4.

30. Trautmann, L. et al. (2006) Upregulation of PD-1 expression on HIV-specific CD8+ T cells leads to reversible immune dysfunction. Nat Med 12 (10), 1198-202.

31. Pauken, K.E. et al. (2016) Epigenetic stability of exhausted T cells limits durability of reinvigoration by PD-1 blockade. Science 354 (6316), 1160-1165.

32. Nussbaum, A.K. et al. (2005) Immunoproteasome-deficient mice mount largely normal CD8+ T cell responses to lymphocytic choriomeningitis virus infection and DNA vaccination. J Immunol 175 (2), 115360.

33. Thomas, S. et al. (2007) Antigen presentation by nonhemopoietic cells amplifies clonal expansion of effector CD8 T cells in a pathogen-specific manner. J Immunol 178 (9), 5802-11.

34. Wherry, E.J. and Kurachi, M. (2015) Molecular and cellular insights into T cell exhaustion. Nat Rev Immunol 15 (8), 486-99.

35. Gupta, P.K. et al. (2015) CD39 Expression Identifies Terminally Exhausted CD8+ T Cells. PLoS Pathog 11 (10), e1005177.

36. Wherry, E.J. et al. (2007) Molecular signature of CD8+ T cell exhaustion during chronic viral infection. Immunity 27 (4), 670-84.

37. Angelosanto, J.M. et al. (2012) Progressive loss of memory T cell potential and commitment to exhaustion during chronic viral infection. J Virol 86 (15), 8161-70.

38. Blackburn, S.D. et al. (2008) Selective expansion of a subset of exhausted CD8 T cells by alphaPD-L1 blockade. Proc Natl Acad Sci U S A 105 (39), 15016-21.

39. Paley, M.A. et al. (2012) Progenitor and terminal subsets of CD8+ T cells cooperate to contain chronic viral infection. Science 338 (6111), 1220-5.

40. Zehn, D. et al. (2016) Immune-surveillance through exhausted effector T-cells. Curr Opin Virol 16, 4954.

41. McKinney, E.F. et al. (2015) T-cell exhaustion, co-stimulation and clinical outcome in autoimmunity and infection. Nature 523 (7562), 612-6.

42. Klenerman, P. and Oxenius, A. (2016) T cell responses to cytomegalovirus. Nat Rev Immunol 16 (6), 367-77.

43. Lelic, A. et al. (2012) The polyfunctionality of human memory CD8+ T cells elicited by acute and chronic virus infections is not influenced by age. PLoS Pathog 8 (12), e1003076.

44. Cicin-Sain, L. et al. (2011) Cytomegalovirus-specific T cell immunity is maintained in immunosenescent rhesus macaques. J Immunol 187 (4), 1722-32.

45. Polic, B. et al. (1998) Hierarchical and redundant lymphocyte subset control precludes cytomegalovirus replication during latent infection. J Exp Med 188 (6), 1047-54.

46. Taddei, T.H. et al. (2016) HIV, Aging, and Viral Coinfections: Taking the Long View. Curr HIV/AIDS Rep $13(5), 269-78$.

47. Hansen, S.G. et al. (2013) Cytomegalovirus vectors violate CD8+ T cell epitope recognition paradigms. Science 340 (6135), 1237874. 
514

515

516

517

518

519

520

521

522

523

524

525

526

527

528

529

530

531

532

533

534

535

536

537

538

539

540

541

542

543

544

545

546

547

548

549

550

551

552

553

554

555

556

557

558

559

560

561

562

48. Hansen, S.G. et al. (2016) Broadly targeted CD8(+) T cell responses restricted by major histocompatibility complex E. Science 351 (6274), 714-20.

49. Chang, W.L. and Barry, P.A. (2003) Cloning of the full-length rhesus cytomegalovirus genome as an infectious and self-excisable bacterial artificial chromosome for analysis of viral pathogenesis. J Virol 77 (9), 5073-83.

50. Murray, S.E. et al. (2017) Fibroblast-adapted human CMV vaccines elicit predominantly conventional CD8 T cell responses in humans. J Exp Med 214 (7), 1889-1899.

51. Holtappels, R. et al. (2000) Enrichment of immediate-early 1 (m123/pp89) peptide-specific CD8 T cells in a pulmonary $\mathrm{CD} 62 \mathrm{~L}(\mathrm{lo})$ memory-effector cell pool during latent murine cytomegalovirus infection of the lungs. J Virol 74 (24), 11495-503.

52. Karrer, U. et al. (2003) Memory inflation: continuous accumulation of antiviral CD8+ T cells over time. $\mathrm{J}$ Immunol 170 (4), 2022-9.

53. Seckert, C.K. et al. (2012) Viral latency drives 'memory inflation': a unifying hypothesis linking two hallmarks of cytomegalovirus infection. Med Microbiol Immunol 201 (4), 551-66.

54. Alexandre, Y.O. et al. (2014) Deciphering the role of DC subsets in MCMV infection to better understand immune protection against viral infections. Front Microbiol 5, 378.

55. Cicin-Sain, L. et al. (2012) Cytomegalovirus infection impairs immune responses and accentuates Tcell pool changes observed in mice with aging. PLoS Pathog 8 (8), e1002849.

56. Munks, M.W. et al. (2006) Four distinct patterns of memory CD8 T cell responses to chronic murine cytomegalovirus infection. J Immunol 177 (1), 450-8.

57. Munks, M.W. et al. (2006) Genome-wide analysis reveals a highly diverse CD8 T cell response to murine cytomegalovirus. J Immunol 176 (6), 3760-6.

58. Snyder, C.M. et al. (2008) Memory Inflation during Chronic Viral Infection Is Maintained by Continuous Production of Short-Lived, Functional T Cells. Immunity 29 (4), 650-659.

59. O'Hara, G.A. et al. (2012) Memory T cell inflation: understanding cause and effect. Trends Immunol $33(2), 84-90$.

60. Sierro, S. et al. (2005) Evolution of diverse antiviral CD8+ T cell populations after murine cytomegalovirus infection. Eur J Immunol 35 (4), 1113-23.

61. Torti, N. et al. (2011) Non-hematopoietic cells in lymph nodes drive memory CD8 T cell inflation during murine cytomegalovirus infection. PLoS Pathog 7 (10), e1002313.

62. Seckert, C.K. et al. (2011) Antigen-presenting cells of haematopoietic origin prime cytomegalovirusspecific CD8 T-cells but are not sufficient for driving memory inflation during viral latency. Journal of General Virology 92 (9), 1994-2005.

63. Hutchinson, S. et al. (2011) A dominant role for the immunoproteasome in CD8+ T cell responses to murine cytomegalovirus. PLoS One 6 (2), e14646.

64. Dekhtiarenko, I. et al. (2016) Peptide Processing Is Critical for T-Cell Memory Inflation and May Be Optimized to Improve Immune Protection by CMV-Based Vaccine Vectors. PLoS Pathog 12 (12), e1006072.

65. Kurz, S.K. et al. (1999) Focal transcriptional activity of murine cytomegalovirus during latency in the lungs. J Virol 73 (1), 482-94.

66. Seckert, C.K. et al. (2009) Liver sinusoidal endothelial cells are a site of murine cytomegalovirus latency and reactivation. J Virol 83 (17), 8869-84.

67. Simon, C.O. et al. (2006) CD8 T cells control cytomegalovirus latency by epitope-specific sensing of transcriptional reactivation. J Virol 80 (21), 10436-56.

68. Dekhtiarenko, l. et al. (2013) The context of gene expression defines the immunodominance hierarchy of cytomegalovirus antigens. J Immunol 190 (7), 3399-409.

69. Farrington, L.A. et al. (2013) Competition for antigen at the level of the APC is a major determinant of immunodominance during memory inflation in murine cytomegalovirus infection. J Immunol 190 (7), 3410-6. 
70. Borkner, L. et al. (2017) Immune Protection by a Cytomegalovirus Vaccine Vector Expressing a Single Low-Avidity Epitope. J Immunol 199 (5), 1737-1747.

71. Boppana, S.B. and Britt, W.J. (1996) Recognition of human cytomegalovirus gene products by HCMVspecific cytotoxic T cells. Virology 222 (1), 293-6.

72. Komatsu, H. et al. (2003) Population analysis of antiviral T cell responses using MHC class I-peptide tetramers. Clin Exp Immunol 134 (1), 9-12.

73. Hahn, G. et al. (1998) Cytomegalovirus remains latent in a common precursor of dendritic and myeloid cells. Proceedings of the National Academy of Sciences 95 (7), 3937-3942.

74. Kemball, C.C. et al. (2005) Late priming and variability of epitope-specific CD8+ T cell responses during a persistent virus infection. J Immunol 174 (12), 7950-60.

75. Vezys, V. et al. (2006) Continuous recruitment of naive T cells contributes to heterogeneity of antiviral CD8 T cells during persistent infection. J Exp Med 203 (10), 2263-9.

76. Richter, K. et al. (2012) Antigen amount dictates CD8+ T-cell exhaustion during chronic viral infection irrespective of the type of antigen presenting cell. Eur J Immunol 42 (9), 2290-304.

77. Prober, C.G. (2012) 204 - Herpes Simplex Virus A2 - Long, Sarah S. In Principles and Practice of Pediatric Infectious Diseases (Fourth Edition), pp. 1026-1035.e3, Content Repository Only!

78. Grzimek, N.K. et al. (2001) Random, asynchronous, and asymmetric transcriptional activity of enhancer-flanking major immediate-early genes ie $1 / 3$ and ie2 during murine cytomegalovirus latency in the lungs. J Virol 75 (6), 2692-705.

79. Nakamoto, Y. and Kaneko, S. (2003) Mechanisms of viral hepatitis induced liver injury. Curr Mol Med $3(6), 537-44$.

80. Bergthaler, A. et al. (2010) Viral replicative capacity is the primary determinant of lymphocytic choriomeningitis virus persistence and immunosuppression. Proc Natl Acad Sci U S A 107 (50), 21641-6. 81. Tay, S.S. et al. (2014) Antigen expression level threshold tunes the fate of CD8 T cells during primary hepatic immune responses. Proc Natl Acad Sci U S A 111 (25), E2540-9.

82. Utzschneider, D.T. et al. (2016) High antigen levels induce an exhausted phenotype in a chronic infection without impairing T cell expansion and survival. J Exp Med 213 (9), 1819-34.

83. Reddehase, M.J. et al. (1994) The conditions of primary infection define the load of latent viral genome in organs and the risk of recurrent cytomegalovirus disease. J Exp Med 179 (1), 185-93. 84. Redeker, A. et al. (2014) Viral inoculum dose impacts memory T-cell inflation. Eur J Immunol 44 (4), 1046-57.

85. Pollock, J.L. and Virgin, H.W.t. (1995) Latency, without persistence, of murine cytomegalovirus in the spleen and kidney. J Virol 69 (3), 1762-8.

86. Wong, Y.C. et al. (2015) Immune outcomes in the liver: Is CD8 T cell fate determined by the environment? J Hepatol 63 (4), 1005-14.

87. Mueller, S.N. et al. (2007) Viral targeting of fibroblastic reticular cells contributes to immunosuppression and persistence during chronic infection. Proc Natl Acad Sci U S A 104 (39), 15430-5. 88. Welten, S.P. et al. (2015) The viral context instructs the redundancy of costimulatory pathways in driving CD8(+) T cell expansion. Elife 4. doi: 10.7554/eLife.07486

89. Xu, H.C. et al. (2014) Type I interferon protects antiviral CD8+ T cells from NK cell cytotoxicity. Immunity 40 (6), 949-60.

90. Crouse, J. et al. (2014) Type I interferons protect T cells against NK cell attack mediated by the activating receptor NCR1. Immunity 40 (6), 961-73.

91. Teijaro, J.R. et al. (2013) Persistent LCMV infection is controlled by blockade of type I interferon signaling. Science 340 (6129), 207-11.

92. Wilson, E.B. et al. (2013) Blockade of chronic type I interferon signaling to control persistent LCMV infection. Science 340 (6129), 202-7.

93. Dag, F. et al. (2014) Reversible silencing of cytomegalovirus genomes by type I interferon governs virus latency. PLoS Pathog 10 (2), e1003962. 
94. Aubert, R.D. et al. (2011) Antigen-specific CD4 T-cell help rescues exhausted CD8 T cells during chronic viral infection. Proc Natl Acad Sci U S A 108 (52), 21182-7.

614 95. Matloubian, M. et al. (1994) CD4+ T cells are required to sustain CD8+ cytotoxic T-cell responses 615 during chronic viral infection. J Virol 68 (12), 8056-63.

616 96. Waggoner, S.N. et al. (2011) Natural killer cells act as rheostats modulating antiviral T cells. Nature 617481 (7381), 394-8.

618 97. Humphreys, I.R. et al. (2007) OX40 costimulation promotes persistence of cytomegalovirus-specific CD8 T Cells: A CD4-dependent mechanism. J Immunol 179 (4), 2195-202.

98. Snyder, C.M. et al. (2009) CD4+ T cell help has an epitope-dependent impact on CD8+ T cell memory inflation during murine cytomegalovirus infection. J Immunol 183 (6), 3932-41.

99. Walton, S.M. et al. (2011) T-cell help permits memory CD8(+) T-cell inflation during cytomegalovirus latency. Eur J Immunol 41 (8), 2248-59.

100. Jones, M. et al. (2010) IL-10 restricts memory T cell inflation during cytomegalovirus infection. J Immunol 185 (6), 3583-92.

101. Almanan, M. et al. (2017) Tissue-specific control of latent CMV reactivation by regulatory T cells. PLoS Pathog 13 (8), e1006507.

102. Rochman, Y. et al. (2009) New insights into the regulation of T cells by gamma(c) family cytokines. Nat Rev Immunol 9 (7), 480-90.

103. Bachmann, M.F. et al. (2007) Differential role of IL-2R signaling for CD8+ T cell responses in acute and chronic viral infections. Eur J Immunol 37 (6), 1502-12.

104. Redeker, A. et al. (2015) The Quantity of Autocrine IL-2 Governs the Expansion Potential of CD8+ T Cells. J Immunol 195 (10), 4792-801.

105. Beltra, J.C. et al. (2016) IL2Rbeta-dependent signals drive terminal exhaustion and suppress memory development during chronic viral infection. Proc Natl Acad Sci U S A 113 (37), E5444-53.

106. Nanjappa, S.G. et al. (2011) Immunotherapeutic effects of IL-7 during a chronic viral infection in mice. Blood 117 (19), 5123-32.

107. Pellegrini, M. et al. (2011) IL-7 engages multiple mechanisms to overcome chronic viral infection and limit organ pathology. Cell 144 (4), 601-13.

108. Presti, R.M. et al. (1998) Interferon gamma regulates acute and latent murine cytomegalovirus infection and chronic disease of the great vessels. J Exp Med 188 (3), 577-88.

109. Ou, R. et al. (2001) Critical role for alpha/beta and gamma interferons in persistence of lymphocytic choriomeningitis virus by clonal exhaustion of cytotoxic T cells. J Virol 75 (18), 8407-23.

110. Moskophidis, D. et al. (1994) Resistance of lymphocytic choriomeningitis virus to alpha/beta interferon and to gamma interferon. J Virol 68 (3), 1951-5.

111. Yoon, H. et al. (2010) The cell cycle time of CD8+ T cells responding in vivo is controlled by the type of antigenic stimulus. PLoS One 5 (11), e15423. 